\title{
Análise do nível de ansiedade pré-competitiva em atletas juvenis e adultos praticantes de Muaythai \\ Analysis of the level of pre-competitive anxiety in young and adult athletes practitioners of Muaythai \\ Análisis del nivel de ansiedad precompetitiva en atletas jóvenes y adultos practicantes de Muaythai \\ * Luana Caroline Contessoto, *Luciane CristinaArantes da Costa, ***Ana Luíza BarbosaAnversa, ** Luciana Boligon Refundini \\ *U niversidade Estadual de Maringá (Brasil), ${ }^{* *}$ Centro U niversitário M etropolitano de Maringá (Brasil)
}

Resumo. A ansiedade no contexto esportivo pode provocar emoções que, quando negativas, poderão influenciar 0 desempenho de atletas. 0 objetivo deste estudo foi analisar o nível de ansiedade pré-competitiva de atletas juvenis e adul tos praticantes de Muaythai presentes no 1을 Campeonato Paranaense de Muaythai. A amostra foi composta por 99 atletas, de ambos os sexos, com nível técnico de amador a avançado eidade médiade 23,04 ( $\pm 7,53)$ (14 a51 anos). Como instrumento de coleta, foi utilizado o questionário CSAI-2R. Para comparação entre os sexos utilizou-se o U M ann-Whitney, paraacomparação das categorias o Kruskal-Wallis e para verificar as múltiplas comparações foi utilizado o teste deT ukey. A análise estatística foi realizada por meio do programaSPSS 15. 0. O s resultadosobtidosindicam que as atletas do sexo feminino são mais autoconfiantes que os atletas do sexo masculino ( $p=.043)$. Em relação à idade, os atletas juvenis apresentaram maior ansiedade cognitiva se comparados aos atletas adultos ( $p=.023$ ). Conclui-se que: entre os participantes do campeonato de M uaythai, as mulheres são mais autoconfiantes que os homens e os atletas da categorial juvenil são mais ansiosos cognitivamente que os adultos.

Palavras-chave: Lutas; Transtornos de ansiedade; Atletas; M uaythai; Competição; Autoconfiança.

\begin{abstract}
Anxiety in the sports context can cause emotions that, when negative, can influence the performance of athletes. The aim of this study was to analyze the level of pre-competitive anxiety of young and adult athletes practitioners of Muaythai present in the 1st M uaythai Parana Championship. A sample consisted of 99 athletes, of both sexes, with technical level from amateur to advanced and average age of $23,04( \pm 7,53)$ (14 to 51 years). As the collection instrument, the CSAI-2R questionnaire was used. To compare the sexes the $U$ M ann-W hitney was used, to compare categories the K ruskal-Wallis was used and to verify the multiple comparisons theTukey test was used. The statistical analysis was performed using the SPSS 15.0 program. The results obtained indicate that female athletes are more self-confident than male athletes $(p=.043)$. In relation to age, young athletes showed greater cognitive anxiety when compared to adult athletes $(p=.023)$. It is concluded that: among the participants in the M uaythai championship, women are more self-confident than men and young athletes are more cognitively anxious than adults.
\end{abstract}

Keywords: Fight; Anxiety disorders; Athletes; Muaythai; Competition; Self-confidence.

Resumen. La ansiedad en el contexto deportivo puede provocar emociones que, cuando son negativas, pueden influir en el rendimiento de los atletas. El objetivo de este estudio fue analizar el nivel de ansiedad precompetitiva de los atletas jóvenes y adultos practicantes de M uaythai presentes en el ler Campeonato Paranaense de Muaythai. L La muestra estaba compuesta por 99 atletas, de ambos sexos, con un nivel técnico desde amateur hasta avanzado y una edad media de 23,04 ( \pm 7,53) (14 a51 años). Se utilizó como instrumento de recolección el cuestionario CSAI-2R. Para la comparación entre los sexos, se usó el U M ann-W hitney, para la comparación de las categorías K ruskal-Wallis y para verificar las comparaciones múltiples se usó la prueba deTukey. El análisis estadístico se realizó utilizando el programa SPSS 15.0. Los resultados obtenidos indican que las atletas femeninas tienen más confianza en sí mismas que los atletas masculinos ( $p=.043$ ). En relación con a la edad, los atletas jóvenes presentaron mayor ansiedad cognitiva en comparación con los atletas adultos ( $p=.023)$. Se concluye que: entre los participantes del campeonato de Muaythai, las mujeres tienen más confianza en sí mismas que los hombres y los atletas de la categoría juvenil tienen más ansiedad cognitiva que los adultos.

Palabras-clave: Lucha; Trastornos de ansiedad;Atletas; Muaythai; Competición; Autoconfianza.

Fecharecepción: 06-05-20. Fechade aceptación: 29-10-20

Luana Caroline Contessoto

contessoto15@ hotmail.com 


\section{Introdução}

A ansiedade no contexto esportivo pode ser um fator benéfico ou prejudicial no desempenho do atleta de acordo com o modo que este interpreta ou reage frente aos seus sintomas. Se 0 atleta detecta a ansiedade como um desafio ou uma meta a ser alcançada ela se tornará um agente facilitador para seu rendimento, no entanto, se a entende como negativa passa a apresentar sintomas de medo, nervosismo, insegurança, preocupação frente à competição e esses sentimentos certamente atuarão como agente prejudicial para 0 atleta, seu rendimento e resultado (Weinberg $\&$ Gould, 2008; Souza, Teixeira \& Lobato, 2012; Interdonato, Miarka\& Franchini, 2013). Frente a esse quadro, o maior desafio no contexto esportivo é encontrar o nível de ansiedade ótimo.

De acordo com ateoria do «U» invertido (Weinberg $\&$ Gould, 2008) determinadas modalidades requerem um ponto ótimo de ativação, podendo variar desde a ausênciatotal de excitação atéa excitação extrema, assim 0 atleta poderá encontrar-se em um estado que 0 desanimarácompletamente ou que o ativaráem excesso, sendo ambos prejudiciais à sua atuação. Deste modo, 0 nível ótimo de ativação é aquele em que 0 atleta se encontra ativado (estimulado) de maneira ideal, tanto física quanto psicologicamente, para competir.

Se tratando do Muaythai, foco do presente estudo, normalmente os combates são de três minutos por um minuto de descanso, e nestes os lutadores tentam nocautear seu oponente ou realizar o maior número de golpes, bem como se defender dos golpes desferidos pelo adversário. Por este motivo, esportes de combate, como este, que necessitam de velocidade, potência, resistência e força requerem um elevado nível de ativação por meio da ansiedade somática (alterações fisiológicas percebidas tais como, aumento dafrequência cardíaca, sudorese, tensão muscular, entre outros), isto é, 0 atleta precisa responder com golpes rápidos e precisos, em um curto espaço de tempo, para al cançar o nocaute ou o maior somatório de pontos durante a luta(O liveria, Areas-N eto, M orales \& Calomeni, 2014).

Deste modo, se 0 atleta apresentar níveis muito baixos de ansiedade ele poderá entrar no combate relaxado demais, ou então, se tiver níveis acima do ideal poderá iniciar 0 combate ansioso 0 suficiente e levar um golpe que pode atélevá-lo ao nocaute. Assim, deixar um indivíduo ativado é deixá-lo excitável tanto física quanto mentalmente para qualquer ação, oferecendo condições para que ele real ize sua melhor atuação sem estimulálo a um grau que possa prejudicálo (Sofia \&
Cruz, 2013; Campos \& Pontes, 2015).

Estudos têm evidenciado que a competição pode ser um ambiente interpretado pelamaioriados atletas como algo ameaçador, de acordo com os agentes estressores internos e externos, podendo variar em relação à idade, sexo, tipo de modalidade, pressão social, vitória, entre outros aspectos (Souza, Teixeira \& Lobato, 2012). Essa maneira negativa que os atletas interpretam esse ambiente, pode prejudicá-los durante a competição (Fatma, Ketin \& Üstün, 2010).

Nos estudos que relacionam ansiedade e lutas, os achados apontam que o nível de ansiedade é maior em mulheres (Tineli, Rover, Fin, Nodari--Júnior, Fiedler \& Baretta, 2011; Interdonato, Miarka \& Franchini, 2013; Sofia \& Cruz, 2013; Verdarguer, Más, Rámon \& Conti, 2017; Rocha \& 0 sório, 2018), em atletas mais jovens (Fernandes \& Silva, 2010; Interdonato, Miarka \& Franchini, 2013; Gaetano, Paloma \& Gaetano, 2015; Rocha \& 0 sório, 2018) e em atletas com menos experiência na modalidade (Rocha \& 0 sório, 2018; Cevahircioðlu, Çakici \& Durgutluoðlu, 2019). Em relação ao M uaythai, notase que, a modalidade tem se expandindo rapidamenteno âmbito nacional, no entanto, os estudos científicos não acompanharam este aumento, uma vez que são poucos os estudos encontrados sobre a modalidade e, em sua maioria, versam sobre questões comportamentais, benefícios da prática, postura, avaliação física, simulação de controle e utilização de pés (Santos \& Da Veiga, 2012; M ortatti, Cardoso \& Puggina, 2013; Campos \& Pontes, 2015; Delvecchio, Silva \& Farias, 2015), sendo escassos os estudos que relacionam fatores psicológicos, tais como a ansiedade e a modalidade. 0 que se tem sobre as relações de fatores psicológicos e lutas fazem referência ao jiu-jitsu, Judô e Kung Fu (Silva, 2008; Bernardi, 2009), não sendo identificadas questões específicas do Muaythai, 0 que remete a importância do presente artigo.

Diante disso, este estudo tem como objetivo analisar o nível de ansiedade précompetitiva em atletas juvenis e adultos praticantes de Muaythai, verificando se há diferenças entre sexo e idade e buscando apontar estratégias para o controle dos níveis elevados de ansiedade momentos antes da competição.

\section{M ateriais e M étodo}

A pesquisa se caracteriza como quantitativa do tipo descritivo inferencial, que de acordo com Thomas, $\mathrm{N}$ elson e Silverman (2012) busca identificar as características principais do objeto de estudo por meio 
de observação, registro, análise e descrição objetivas e completas dos fatos sem a manipulação dos mesmos.

Para o levantamento dos artigos na literatura que deram sustentação as discussões dos achados empíricos, realizou-se uma busca nas seguintes bases de dados: LILACS, M edline e Google Scholar. Foram utilizados para buscados artigosos seguintes descritores (tanto nalíngua portuguesa quanto inglesa): «M uaythai», «M uaithai», «M uaythay», «atletas», «ansiedade», «ansiedade précompetitiva», «artes marciais» e «lutas».

Os critérios de inclusão dos artigos foram: artigos publicados em português, inglês e espanhol e que retratassem a temática referente à ansiedade tanto em atletas jovens e/ ou adultos, masculino e feminino, modalidades individuais ou coletivas e lutas.

\section{Participantes}

Participaram do estudo atletas do 10 Campeonato Paranaense de M uaythai. A população foi composta de 170 atletas de Muaythai e a amostra foi constituída por 99 atletas, selecionados de forma aleatória, das cidades de Cascavel, Ivaiporã, Londrina, Mandaguari, Maringá e Paranavaí, de ambos os sexos e com idade média de 23,04 ( \pm 7,53) (14 a51 anos). 0 scritérios paraainclusão dos atletas selecionados para o estudo foram: (a) ter no mínimo 3 meses de treino; (b) os atletas menores de idade tinham que estar participando da categoriajuvenil (que envolve atletas de 14 a 17 anos de idade), tanto na categoria amadora (até 10 lutas) quanto na avançada (acima de 10 lutas); (c) os atletas adultos (maiores de 18 anos) poderiam estar participando tanto da categoria amadora (até 10 lutas) quanto da avançada (acima de 10 lutas); (d) ser representante do estado do Paraná; (e) aceitar participar da pesquisa; (f) assinar o termo de consentimento livre e esclarecido; (g) foram excluídos da pesquisa aqueles que tinham menos que 3 meses de treino.

\section{Instrumentos}

Para verificar o perfil dos atletas, foi utilizada uma ficha de identificação com dados sócios demográficos. 0 número de lutas apontadas pelos atletas foi utilizado para apresentar as categorias dos mesmos. A categoria amadora representava os atletas que tinham de zero até 10 lutas e a categoria avançada aqueles que tinham mais que 10 lutas.

A análise do nível de ansiedade dos atletas foi feita por meio do CSAI-2R (Cox, Martens \& Russell, 2003) uma versão revisada do CSAI-2 (Competitive State Anxiety Inventory-2) original de Martens, Burdon, Vealey, Bump e Smith (1990) que avalia a ansiedade estado no esporte englobando 17 itens, sendo: ansiedade cognitiva (5), ansiedadesomática(7) eautoconfiança(5) as quaispodem ser respondidas numa escala tipo likert de 1 a 4 pontos, sendo 1 = nada e $4=$ muito.

\section{Procedimentos na coleta de dados}

Primeiramente, foi entregue aos treinadores das equipes uma carta convite para participação no estudo e 0 termo de consentimento livre e esclarecido para todos os atletas adultos entrevistados e para os pais el ou responsáveis dos atletas menores de idade. Todos participaram voluntariamente da pesquisa. A coleta foi real izada no momento da pesagem do campeonato, uma hora antes do início da primeira luta, no próprio local da competição. 0 s próprios atletas preencheram 0 questionário. 0 tempo médio para preenchimento foi de aproximadamente 20 minutos. Por fim, destacase que 0 estudo foi aprovado pelo Comitê de Ética em Pesquisa do Centro Universitário de MaringáUnicesumar sob Parecer 2.042.889.

\section{Análise estatística}

O s dados não foram considerados normais, por isso, se utilizou mediana e intervalo interquartil. Para comparação entre os sexos utilizou-se o teste $U$ MannWhitney e para a comparação das categorias o KruskalWallis. Para ver as múltiplas comparações o teste de Tukey. A análise estatística foi real izada mediante o pacote computadorizado SPSS (Statistical Package for the Social Sciences) 15.0 e foi aceito o nível de significância de $p<.05$.

\section{Resultados}

Em um primeiro momento buscou-se traçar o perfil dos praticantes de M uaythai (sexo, idade, cidade, tempo de prática, frequência semanal e categoria) que participaram do $1^{\circ}$ C ampeonato Paranzense de M uaythai.

Conformeatabela 1, observa-se que o maior número de participantes do estudo era do sexo masculino (78,2\%), os atletas possuíam idade entre 18 a 39 anos (71,7\%), residentesnacidade de $M$ aringá $(48,4 \%)$, com tempo de prática entre 3 mesesa 1 ano $(46,5 \%)$, treinam $5 x$ ou mais durante a semana $(43,4 \%)$ e possuem menos que 10 lutas (adulto amador com $61,6 \%$, seguido do juvenil amador com 26,3\%).

Ao realizar a comparação de ansiedade dos atletas do campeonato em relação ao sexo (tabela 2), pode-se perceber que a autoconfiança foi 0 item que apresentou 


\begin{tabular}{|c|c|c|c|}
\hline & & $\%$ & $\mathrm{n}$ \\
\hline \multirow[t]{2}{*}{ Sexo } & Masculino & 78,2 & 78 \\
\hline & Feminino & 21,8 & 21 \\
\hline \multirow[t]{3}{*}{ Idade } & 14 a 17 anos (juvenil) & 26,2 & 26 \\
\hline & 18 a 39 anos (adulto) & 71,7 & 71 \\
\hline & $=40$ anos (máster) & 2,0 & 2 \\
\hline \multirow[t]{6}{*}{ Cidade } & Maringá & 48,4 & 48 \\
\hline & Londrina & 21,2 & 21 \\
\hline & Mandaguari & 15,1 & 15 \\
\hline & Ivaiporã & 8,1 & 8 \\
\hline & Paranavaí & 5,0 & 5 \\
\hline & Cascavel & 2,0 & 2 \\
\hline \multirow[t]{3}{*}{ Tempo de prática } & 3 meses a 1 ano & 46,5 & 46 \\
\hline & $>1$ ano a 3 anos & 36,4 & 36 \\
\hline & $>3$ anos & 17,2 & 17 \\
\hline \multirow[t]{3}{*}{ Frequência de treino semanal } & $2 x$ na semana & 16,2 & 16 \\
\hline & 3 e $4 x$ na semana & 40,4 & 40 \\
\hline & $5 x$ na semana ou mais & 43,4 & 43 \\
\hline \multirow[t]{4}{*}{ Categoria } & Juvenil Amador & 26,3 & 26 \\
\hline & Juvenil Avançado & 1,0 & 1 \\
\hline & Adulto A mador & 61,6 & 61 \\
\hline & Adulto Avançado & 11,1 & 11 \\
\hline
\end{tabular}

Tabela 2.

Comparação da ansiedade entre os sexos dos atletas participantes do 10 Campeonato Paranaense de Muaythai.

\begin{tabular}{lccccc}
\hline & \multicolumn{2}{c}{ Masculino } & \multicolumn{2}{c}{ Feminino } & $P$ \\
\cline { 2 - 5 } & Mediana & $(\mathrm{Q} 1 ; \mathrm{Q} 3)$ & Mediana & $(\mathrm{Q} 1 ; 03)$ & \\
\cline { 2 - 5 } Ansiedade Cognitiva & 2,00 & $(1,40 ; 2,40)$ & 2,20 & $(1,70 ; 2,60)$ & .271 \\
Ansiedade Somática & 1,75 & $(1,41 ; 2,14)$ & 1,85 & $(1,42 ; 2,28)$ & .447 \\
Autoconfiança & 2,80 & $(2,20 ; 3,40)$ & 3,00 & $(2,60 ; 3,60)$ & $.043^{*}$ \\
\hline * p =.05 Legenda: valores de ansiedade cognitiva e somática e autoconfiança entre homens e
\end{tabular}
mulheres. Fonte: os autores

Tabela 3.

Comparação entre os níveis de ansiedade nas categorias juvenis e adul tos dos atletas participantes

do $1^{\circ}$ Campeonato Paranaense de Muaythai. Juvenil Amador Adulto Amador Adulto Avançado $P$ \begin{tabular}{lccccccc} 
& \multicolumn{1}{c}{ Mediana } & $(\mathrm{Q} 1 ; \mathrm{Q} 3)$ & Mediana & $(\mathrm{Q} 1 ; \mathrm{Q} 3)$ & Mediana & $(\mathrm{Q} 1 ; \mathrm{Q} 3)$ \\
\hline Ansiedade Cognitiva & 2,30 & $(2,00 ; 2,60)$ & 1,80 & $(1,40 ; 2,40)$ & 1,80 & $(1,40 ; 2,50)$ & $\mathbf{0 0 2} \mathbf{3}^{*}$ \\
Ansiedade Somática & 1,85 & $(1,57 ; 2,28)$ & 1,71 & $(1,42 ; 2,14)$ & 1,57 & $(1,35 ; 2,35)$ & 853
\end{tabular} $\begin{array}{llllllll}\text { Ansiedade Somática } & 1,85 & (1,57 ; 2,28) & 1,71 & (1,42 ; 2,14) & 1,57 & (1,35 ; 2,35) & .853 \\ \text { Auto } & 2,80 & (2,20 ; 3,40) & 3,00 & (2,40 ; 3,40) & 2,40 & (2,10 ; 3,00) & 149\end{array}$

* $p=.05$. Legenda: val ores de ansiedade cognitiva e somática e autoconfiança em atletas juvenis e adultos. Fonte: os autores.

índices mais elevados se comparado àansiedade cognitiva e somática em ambos os sexos.

Encontrou-se diferença significativa $(p=.043) \mathrm{em}$ relação ao sexo, mostrando que as atletas do sexo feminino são mais autoconfiantes que os do sexo masculino. 0 utro fator de comparação analisado foram os níveis de ansiedade entre os atletas das categorias juvenis e adultos (tabela 3).

Ao comparar os níveis de ansiedade nas categorias juvenise adultos, constatou-se que a ansiedade cognitiva obteve diferença significativa $(p=.023)$ para os atletas juvenis amadores quando comparados aos adultos amadores. Na comparação da ansiedade somática e na autoconfiança não houve diferença significativa entre os atletas das diferentes categorias (tabela 3).

\section{Discussão}

Os resultados encontrados evidenciaram que os níveis de autoconfiança foram maiores do que os níveis de ansiedade somática e cognitiva, em ambos os sexos. Este resultado corroboracom o de Gomes, Souza, Yoshida eFernandes (2019) queao analisar o impacto queaprática de Jiu-jitsu possui na vida dos atletas, verificou 0 aumento da autoconfiança ao aprenderem um esporte que envolve aspectos defensivos, assim como o M uaythai. 0 utro estudo (Isidoro, Tobal, Escudero, O rtega \& Sanchez, 2014) aponta que devido aos conhecimentos teóricos e filosóficos que envolvem as artes marciais (como no Judô, Karatê e Wushu, que são semelhantes ao Muaythai) e o tipo do esporte (que requer uma elevada atenção e foco na tarefa a cumprir), a prática destas modalidades favorecem o controle dos estados emocionais dos praticantes, tanto em treinamento quanto em competição.

Em relação a ansiedade somática e cognitiva não houve diferenças significativas, mas os níveis foram maiores nas mulheres, o que corrobora comVerdarguer, Más, Rámon e Conti (2017) que ao analisarem atletas masculinosefemininos, tanto demodalidadesindividuais quanto coletivas, encontrou que os níveis da ansiedade somática e cognitiva foram maiores em mulheres.

Por outro lado, o presente estudo encontrou uma diferençasignificativapara aautoconfiança, a qual obteve níveis mais elevados nas atletas mulheres do que em homens, o que contrapõe os achados de outros estudos (Interdonato, Miarka \& Franchini, 2013; Tineli, Rover, Fin, Nodari-Júnior, Fiedler \& Baretta, 2011) que ao analisarem os níveis de ansiedade e a autoconfiança de atletas de Judô, deambosossexos, durante participações em campeonatos e também em um estudo de revisão sistemática (Rocha\& 0 sório, 2018) realizado com atletas de diferentes modalidades esportivas, tanto de esportes individuais quanto coletivos, encontraram que a ansiedade cognitiva e somática foi maior do que a autoconfiança, principalmente nas mulheres.

Acredita-se que os achados desta pesquisa vão de encontro ao de Pessina (2017) que ao estudar a questão de gênero no Muaythai, aponta que a inserção das mulheres na modalidade mostra 0 empoderamento feminino no âmbito esportivo, principalmente em um esporte considerado «masculino», e isso faz com que sua autoestima e autoconfiança aumentem, pois estas têm conquistado cada vez mais seu espaço na sociedade. Além disso, as praticantes de esportes de combate possuem uma maior autonomia corporal e parecem visualizar a luta em si como menos ameaçadoras do que o sexo masculino, desenvolvendo uma melhor autoconfiança.

Q uanto aos níveis de ansiedade entre atletas jovens e adultos, os achados da presente pesquisa mostraram que a ansiedade cognitiva é maior nos jovens se comparados aos adultos. Em relação à faixa etária, são grandes as preocupações com os níveis de ansiedade nos 
atletas jovens, pois nesta fase da vida ocorrem muita tensão e instabilidade emocional, tanto em homens quanto em mulheres, e em situações de competição a ansiedade tende a aumentar quando estes estão em situações de pressão pel o sucesso e pela vitória, afetando assim o seu desempenho esportivo (Santos \& Fonseca, 2014).

Fernandes e Silva (2010) investigaram 110 atletas de Jiu-jitsu, observando que 0 nível de ansiedade cognitiva nos atletas jovens era maior do que nos atletas mais velhos. Este fato corrobora com Gaetano, Pal oma e Gaetano (2015) que ao anal isarem o nível de ansiedade de jovens atletas em diferentes modalidades esportivas, encontraram que nas modalidades individuais, principalmente nas lutas, os mais novos apresentaram maior nível de ansiedade.

Ainda neste contexto, al guns estudos que anal isaram o nível de ansiedade competitiva e pré-competitiva em atletas jovens de outras modalidades, tais como polo aquático e futebol respectivamente, também encontraram que 0 nível de ansiedade é maior neste público (Serrano, Reis, Fonseca, Paulo, Honório \& Batista, 2019; Hernández, Iturriaga, Barquín \& Lara, 2020).

Algumas investigações apontam que quanto mais novo 0 atleta, por consequência, menor a experiência em competições, maior é a manifestação da ansiedade (Souza, Teixeira \& Lobato, 2012; Cevahircioðlu, Çakici \& Durgutluoðlu, 2019), e isto, pode estar relacionado com 0 fato desses atletas serem menos submetidos a situações de ansiedade em competições e também nos treinamentos, enquanto que os atletas mais experientes são submetidos mais vezes a momentos de estresse, tornando maioressuacapacidade de controlar a ansiedade (Interdonato, Miarka\& Franchini, 2013; Rocha\& 0 sório, 2018).

As competições que envolvem atletas da categoria juvenil torna-se um cenário ainda mais propício para 0 aumento da ansiedade, pois é nela que o jovem atleta irácolocar à provatodas assuas habilidades, competências, status social, isto é, suas primeiras experiências esportivas serão exibidas publicamente e isso poderá ser encarado por ele como algo ameaçador, elevando assim seu nível de ansiedade estado se comparado aos atletas adultos, que já possuem uma certa experiência com ambientes competitivos(Campani, Voser, Hernandez \& H aas, 2011).

0 modo como os atletas interpretam os níveis de ansiedade estado podem ser determinantes para o bom desempenho em uma competição, por este motivo é essencial que estratégias de enfrentamento sejam utilizadas pelos atletas, pois estas são maneiras de lidar com eventos estressantes de forma adequada, como a competição, fazendo com que o indivíduo consiga controlar suas emoções e assim diminuir seus níveis de ansiedade, melhorar sua motivação, autoconfiança e desempenho (Belem, Costa, Both, Passos \& Vieria, 2016).

Castro-Sánchez, Zurita-O rtega, Chacón-Cuberos e Lozano-Sánchez (2019) apontam que é preciso que 0 treinador crie um clima motivacional orientado para a tarefa durante os treinamentos, bem como ofereça reforço positivo para que assim seus atletas sejam estimulados a terem um nível mais baixo de ansiedade. Porém, se mesmo assim constatar níveis elevados de ansiedade, é indicado realizar técnicas de relaxamento como meio de reduzir a ansiedade estado.

Torrealva e Bossio (2019) aplicaram três tipos de exercícios da prática de atenção plenaque buscamelhorar o bem-estar psicológico devido a redução dos sintomas ansiosos, em atletas de Karatê, Kung Fu e Taekwondo. Os exercícios aplicados foram a varredura corporal, yoga consciente e atenção plena na respiração, durante seis semanas, totalizando 12 sessões em grupo, com duração de uma hora, duas vezes por semana. 0 grupo experimental após as seis semanas de intervenção teve uma diminuição dos níveis da ansiedade cognitiva e somática, bem como um aumento da autoconfiança se comparados aos participantes do grupo controle, que participaram no máximo de duas sessões. Estas estratégias se demonstraram eficazes no controle da ansiedade dos atletas durantes as competições, o que poderia ser desenvolvido por profissionais capacitados para tal, desde que os treinadores compreendam sua necessidade.

Diante do exposto, constata-se que a ansiedade pode ter influênciano contexto esportivo competitivo, porém essa relação poderá ser positiva ou negativa dependendo da maneira como o atleta encara a competição e, por isso, tanto o momento pré-competitivo quanto a competição em si, devem ser muito bem organizados e planejados para que 0 atleta chegue em boas condições físicas e psicológicas parasuas competições, necessitando de diferentes estratégias para o controle dos aspectos negativos causados pela ansiedade.

\section{Conclusões}

0 presente estudo tinha como objetivo analisar 0 nível de ansiedade pré-competitiva em atletas juvenis e adultos praticantes de Muaythai, verificando se há 
diferenças entre sexo e idade e buscando apontar estratégias para o controle dos níveis elevados de ansiedade momentos antes da competição.

A análise dos níveis de ansiedade nesta investigação permitiu observar que as atletas do sexo feminino, participantes do campeonato analisado, são mais autoconfiantes que os atletas do sexo masculino, indo de encontro aos achados de outros estudos. Este fato nos leva a crer que ao aprenderem uma modal idade de luta, a autoconfiança da mulher é otimizada e elas se sentem mais independentes e seguras, porém outros estudos que venham investigar a temática são fundamentais para que possa ser analisado de forma aprofundada os benefícios da prática de modalidades de combate no público feminino.

Em relação à idade, os atletas juvenis possuem maior ansiedade cognitiva se comparados aos adultos, o que é encontrado em diversos estudos com diversas modalidades, indo ao encontro dos nossos achados. Q uanto maior a idade e maior a experiência, tanto no esporte quanto na vida, 0 atleta parece perceber a competição de forma positiva.

Conclui-se então que a ansiedade pode ter influência no contexto esportivo competitivo, porém essa relação poderá ser positiva ou negativa dependendo da maneira como 0 atl eta encara a competição e da faixa etária dos mesmos. Além disso, os treinadores deveriam investigar as causas que afetam o fracasso dos atletas e juntos procurar amenizá-las, principalmente com os mais jovens, por meio de estratégias que buscam reduzir 0 nível de ansiedade, contribuindo assim com o seu desenvolvimento e desempenho.

A ponta-se que este estudo se limita por trazer a realidade de atletas de uma pequena região do Brasil em um campeonato regional, devido a isso, sugere-se que outros estudos sejam realizados com atletas desta modalidade para que se possa al cançar um público maior e mais heterogêneo e assim, traçar um perfil mais amplo de praticantes para que além de analisar quais são os níveis de ansiedade pré-competitiva, sejam pensadas estratégias de inter venções psicológicas em atletas como as de coping (enfrentamento) para que esses possam controlar aansiedade, aumentar o nível de autoconfiança e assim obterem resultados positivos durante as competições.

\section{Referências}

Belem, I., Costa, L.C.A., Both, J., Passos, P.C.B. \& Vieria, J.L.L. (2016) 0 estresse no MMA: as estratégias de enfrentamento podem melhorar 0 desempenho dos lutadores?. Rev Bras M ed Esporte, 22(4), 287-290. http:/ / dx. doi.org/ 10.1590/ 1517869220162204147514

Bernardi, M. (2009) Nível de ansiedade em atletas de jiujitsu pré competição. Trabalho de conclusão de curso (Graduação em Educação Física) - Universidade de Caxias do Sul, Centro de Filosofia e Educação de Caxias do Sul.

Campani, M.M.,Voser, R.C., Hernandez, J.A.E. \& Haas, A.N. (2011) A ansiedade na iniciação esportiva: estudo de revisão. Lecturas: Educación física y deportes, 16(157), 1.

Campos, W.M . \& Pontes, J.A.M . (2015) Lutas em foco: o M uay Thai e a mudança de comportamento dos alunos da universidade federal do Ceará. Fiep Bulletin online, 85, 1-5. doi: 10.16887/ 85.a1.92

Castro-Sánchez, M., Zurita-O rtega, F., ChacónCuberos, R. \& Lozano-Sánchez, A. M. (2019) Clima motivacional y niveles de ansiedad en futbolistas de categorías inferiores. Retos: nuevas tendencias en educación física, deporte y recreación, 35, 164-169. doi: https:/ / doi.org/ 10.47197/ retos. v0i35.63308 Cevahircioðlu, B., Çakici, H.A. \& Durgutluoðlu, M. (2019)The examination of pre-competition anxiety levels of Muay-thai athletes. I Int Anatolia Sport Sci, 4(2), 7-11. doi: 10.5505/ jiasscience. 2019.92486

Cox, R.H., Martens, M.P. \& Russell, W.D. (2003) Measuring Anxiety in Athletics: The Revised Competitive State Anxiety Inventory-2. Sport \& Exerc Psychology, 25, 519-533.

Delvecchio, F.B., Silva, J.J.R. \& Farias, C.B. (2015) A nál ise temporal de combates de M uay-Thai de nível nacional: efeitos dafase competitiva. RevArtes $M$ arciales Asiát, 10(1), 34-41. doi: 10.18002/ rama.v10i1.1635

Fatma, A., Tekin, M . \& Üstün, E. (2010) The assesment of the failure and success cases of the sportmen participating to muai-thai turkey championship. Rev Science, M ovement and $\mathrm{H}$ ealth, 10 (1), 5-7.

Fernandes, M.G. \& Silva, L.H. (2010) Relação entre ansiedade competitiva e experiência em atletas de jiu-jítsu e surfe. Lecturas: Educación física y deportes, 45(15), 1.

Gaetano, R. , Paloma, F. G. \& Gaetano, A. (2015)Anxiety in theYouth Physical and SportActivity. Mediterranean Journal of Social Sciences, 6(3), 227-230. doi: 10.5901/ mjss. 2015. v6n3s2p227

Gomes, S. T. , Souza, R. L. S. Yoshida, H. M . \& Fernandes, P.T. (2019) Autoestima, autoconfiança e ansiedade em praticantes de Jiu-jitsu. Rev Conexões: Educ. Fís. 
Esporte e Saúde, 17, 1-10. doi:10.5901/ mjss. 2015. v6n3s2p227

Hernández, P.J.B., Iturriaga, F.M.A., Barquin, R.R. \& Lara, E.R. (2020) Análisis de la ansiedad competitiva en jugadores jóvenes de waterpolo en función del género y rendimiento. Retos: nuevas tendencias en educación física, deporte y recreación, 38, 20-25. doi: https: / / doi.org/ 10.47197/ retos. v38i38.73450 Interdonato, G.C., Miarka B. \& Franchini, E. (2013) A nálise da ansiedade pré-competitiva e competitiva de jovens judocas. Rev Artes M arcialesAsiát, 2(8), 471479. doi: 10.18002/ rama. v8i2. 977

Isidoro, S.G., Tobal, F.M ., Escudero, P.M ., O rtega, C.G. \& Sanchez, V.O.C. (2014) Las artes marciales, su origen y actual idad: uma visión enfocada en el Judo, Karate yW ushu. Rev Orientando, 8, 141-174.

Mortatti, A.L., Cardoso, A., Puggina, E. F. \& Costa, R.S. (2013) Efeitos da simulação de combates de Muay Thai na composição corporal e em indicadores gerais de manifestação de força. Rev Conexões, 11(1), 218234.

Oliveira, R., Arêas-N eto, N.T., Morales, A.P. \& Calomeni, M.R. (2014) Influência do estresse précompetitivo no desempenho de lutadores. Rev Persp. online: biol. \& saúde, 14(4), 27-35.

Pessina, J.E. (2017) Gênero no M uayThai: uma lutadentro das Artes M arciais. Trabalho de conclusão de curso (Graduação em Educação Física) - Universidade Estadual Paulista «úlio de M esquita Filho», Instituto de Biociências do Rio Claro.

Rocha, V.V.S. \& O sório. F.L. (2018)Associationsbetween competitive anxiety, athlete characteristics and sport context: evidence from a systematic review and meta-analysis. Rev Arch Clin Psychiatry, 45(3), 67-74. doi:10.1590/ 0101-60830000000160

Santos, G. \& Fonseca, G. M. M. (2014) Nível de ansiedade em jovens atletas de voleibol em situação précompetitiva. Rev Do corpo: Ciências e Artes, 4(1), 1-7.

Santos, R.V. \& Da Veiga, R.A.D.R. (2012) Avaliação postural de praticantes da arte marcial Muaythai no município de Erechim/ RS. Rev Perspectiva, 36(133),
163-178.

Serrano, J., Reis, R., Fonseca, T., Paulo, R., Honório, S. \& Batista, M. (2019) Pre-Competitive Anxiety: a comparative study between «Under 12» and Senior teams in football. Retos: nuevas tendencias en educación física, deporte y recreación, 36, 318-326. doi: https:/ / doi.org/ 10.47197/ retos. v36i36.67129

Silva, R. S. (2008) A ansiedadeesuasinfluênciasno desempenho competitivo de atletas do judô. Trabal ho de concl usão de curso (Graduação em Educação Física) - U niversidade Estadual Paulista, Instituto deBiociênciado Rio Claro. Sofia, R. \& Cruz, J.F. (2013) Relações entre raiva, agressividade, ansiedade e percepção de ameaça na competição desportiva: um estudo em diferentes modalidades de contato físico. RPCD , 13(2), 44-56.

Souza, M.A.P., Teixeira, R.B. \& Lobato, P.L. (2012) $M$ anifestação da ansiedade pré-competitiva em nadadores amadores. Rev Educ Fís UEM , 23(2), 195203. doi: 10.4025/ reveducfis. V23i2. 14737

Tineli, D., Rover, C., Fin, G., Nodari-Júnior, R.J., Fiedler, M. M. \& Baretta, E. (2011) Ansiedade-traço pré-competitiva: um estudo com atl letas dejudô. Rev Unoesc \& Ciência - ACBS, 2(2), 107-116.

Thomas, J. R., N elson, J.K. \& Silverman, S.J. (2012). Métodos de Pesquisa em Atividade Física. 6.ed. Porto Alegre: Artmed.

Torrealva, D.T. \& Bossio, M. R. (2019) Programa basado en mindfulness para la reducción de la ansiedad precompetitiva en desportistas de artes marciales. Retos: nuevas tendencias en educación física, deporte y recreación, 36, 418-426. doi: https:// doi.org/ 10.47197/ retos. v36i36. 66589

Verdaguer, F.J.P., Más, A.G., Ramón, J.C., \& Conti, J.V. (2017) Diferencias de sexo respecto de laansiedad asociada a la competición deportiva. Retos: nuevas tendencias en educación física, deporte y recreación, 31, 193-196. doi: https:// doi. org/ 10.47197/ retos. v0i31. 40325

Weinberg, R.S. \& Gould, D. (2008) Fundamentos da psicologia do esporte e do exercício. 4. ed. Porto Alegre: Artmed.

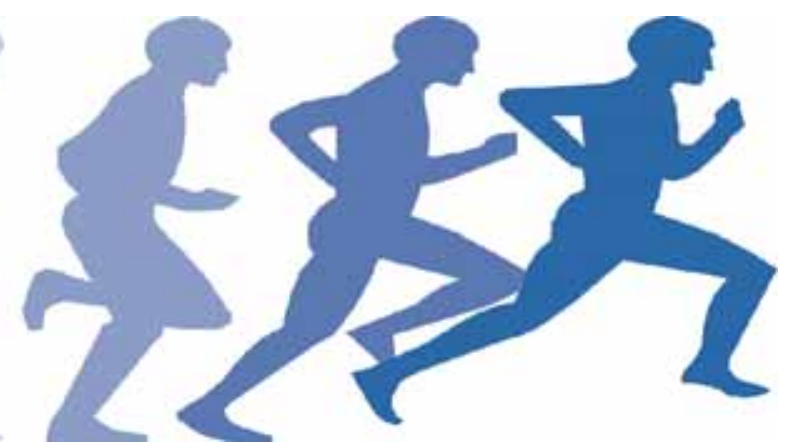

